\title{
Anwendungsfälle und Methoden der künstlichen Intelligenz in der anwendungsorientierten Forschung im Kontext von Industrie 4.0
}

\section{Use cases and methods of artificial intelligence in applied research in an Industry 4.0 context}

\author{
B. Maschler, M.Sc.; D. White, M.Sc.; Prof. Dr.-Ing. Dr. h. c. M. Weyrich, \\ Institut für Automatisierungstechnik und Softwaresysteme (IAS) der Uni- \\ versität Stuttgart, Stuttgart;
}

\section{Schlüsselwörter}

Künstliche Intelligenz (KI), Intelligente Systeme, Datenanalyse, Industrielle Anwendungen, Automatisierungstechnik, Maschinelles Lernen, Fertigungsindustrie, Produktion, Literaturrecherche

\section{Kurzfassung}

Es wird erwartet, dass datengetriebene Methoden künstlicher Intelligenz im Kontext Industrie 4.0 die Zukunft industrieller Fertigung prägen werden. Obwohl das Thema in der Forschung sehr präsent ist, bleibt der Umfang der tatsächlichen Nutzung dieser Methoden unklar. Dieser Beitrag analysiert daher von 2013 bis 2018 veröffentlichte wissenschaftliche Artikel, um statistische Daten über den Einsatz von Methoden künstlicher Intelligenz in der Industrie zu gewinnen. Besonderes Augenmerk wird dabei auf die Trainings- und Evaluations-Datentypen, die Verbreitung in verschiedenen Industriezweigen, die betrachteten Anwendungsfälle sowie die geographische Herkunft dieser Artikel gelegt. Die resultierenden Erkenntnisse werden in praxisnahe Hinweise für Entscheider destilliert.

\section{Einleitung}

Die Bezeichnung „Industrie 4.0“ beschreibt den fortschreitenden Trend der umfassenden Vernetzung von Produktionsanlagen auf der Basis von cyber-physischer Systemen (Kagermann et al. 2013). Diese Entwicklung wird im englischen Sprachraum auch als „Industrial Internet of Things“ beschrieben (Jeschke et al. 2017) und stellt aufgrund der Vielzahl der Kommunikationsteilnehmer und der daraus resultierenden Systemkomplexität erhebliche Anforderungen an die Steuerung derselben und die Verarbeitung der dabei anfallenden Daten (Runkler 2015). 
Eine zentrale Basistechnologie zur Realisierung von Industrie 4.0 sind damit Methoden künstlicher Intelligenz, die einerseits die Komplexität beherrschbar machen und andererseits weitere Mehrwerte realisieren können (Runkler 2015; Sharp et al. 2018; Wang et al. 2018; Muhuri et al. 2019).

Obwohl es seit vielen Jahren eine erhebliche Forschungstätigkeit im Bereich der datengetriebenen Methoden künstliche Intelligenz gibt (Jordan und Mitchell 2015; Sharp et al. 2018; Wang et al. 2018; Muhuri et al. 2019; Diez-Olivan et al. 2019), ist der Umfang deren praktischen Einsatzes in der Industrie nicht gut dokumentiert. Deshalb werden in dieser Veröffentlichung mittels einer Literaturrecherche die Trends hinsichtlich der Nutzung von Methoden künstlicher Intelligenz in verschiedenen Industriesektoren untersucht und dargestellt.

Ausgehend von den genannten derzeitigen Entwicklungen in der Forschung und der Industrie wird in Kapitel 2 der Betrachtungsraum dieser Veröffentlichung näher spezifiziert. Darauf aufbauend wird in Kapitel 3 die genutzte Methodik für die Literaturrecherche beschrieben und die ihr zugrundliegenden Kriterien genannt. Kapitel 4 stellt die resultierenden Ergebnisse vor und gibt neben einem allgemeinen Überblick eine genauere Betrachtung im Hinblick auf die Fertigungsindustrie wider. Kapitel 5 zieht ein Fazit mit besonderem Fokus auf die Auswahl von KIMethoden für Entwicklungsprojekte in der Industrie.

\section{Betrachtungsgegenstand}

Die vorliegende Veröffentlichung betrachtet Anwendungsfälle und Methoden der künstlichen Intelligenz $(\mathrm{KI})$ in der anwendungsorientierten Forschung im Kontext von Industrie 4.0. Die Kernbegriffe dieses Themenfeldes werden im weiteren Verlauf folgendermaßen interpretiert:

- Unter „Methoden der künstlichen Intelligenz“ seien Verfahren zu verstehen, die von Computersystemen verwendet werden, um intelligentes, sonst nur von Menschen gezeigtes Verhalten bei der Lösung spezifischer Aufgaben nachzuahmen (Flasinski 2016). Konkreter müssen diese Verfahren „datengetrieben“ sein und damit auf der automatisierten Informationsbeschaffung aus großen Datenmengen basieren - und nicht auf der Modellierung von zuvor bekannten (physikalischen) Beziehungen durch Menschenhand, wie z.B. in regelbasierten intelligenten Systemen.

- Unter „anwendungsorientierter Forschung“ sei Forschung zu verstehen, die den praktischen Einsatz einer Methode in einem konkreten Geschäftsmodell zur Kostensenkung oder Profitsteigerung zum Ziel hat. Damit sei die Erforschung des Einsatzes einer Methode in einem reinen Forschungskontext ausgeschlossen. 
- Unter „Industrie“ seien Unternehmen zu verstehen, deren Geschäftsmodell auf der Umwandlung von Energie und Materialien zum Zwecke der Herstellung von (Roh-)Materialien, Fertigprodukten oder der Energieversorgung basieren bzw. Dienstleistungen im unmittelbaren Kontext derartiger Geschäftsmodelle anbieten. „Industrie 4.0“ beziehe sich dann auf derartige Unternehmen, die derartige Geschäftsmodelle auf Basis cyberphysischer Produktionssysteme und vernetzter digitaler Prozessketten betreiben (Vogel-Heuser 2017).

\section{Methodik}

Die Methodik der im Folgenden vorgestellten Untersuchung orientiert sich an der Systematic Literature Review (SLM) nach Kitchenham (2004). Ihre Relevanz ergibt sich dabei aus der in Kapitel 1 dargelegten Diskrepanz zwischen den hohen Erwartungen an den Einsatz von KIMethoden in der Industrie und der schlechten Studienlage hinsichtlich dessen tatsächlicher Umsetzung.

Konkret wurde ein 3-stufiger Prozess gewählt: Im Rahmen der Datenerhebung wurden Veröffentlichungen identifiziert, die eine Reihe von Basisanforderungen erfüllen. Diese Veröffentlichungen wurden dann weiterverarbeitet und hinsichtlich zusätzlicher Kriterien manuell gefiltert. Schließlich wurden die verbleibenden Veröffentlichungen manuell analysiert (siehe Abbildung 1).

Die manuelle Filterung und Analyse wurde trotz des Nachteils reduzierter Reproduzierbarkeit einer automatischen Lösung mittels einfacher Suchen oder komplexerer Textanalysetools vorgezogen, da letztere bisher nicht qualitativ vergleichbare Ergebnisse liefern können. Dies liegt in der großen Kontextabhängigkeit der Relevanz genutzter Begriffe sowie der erheblichen inhaltlichen Breite und teilweise durchwachsenen sprachlichen Qualität der betrachteten Veröffentlichungen begründet.

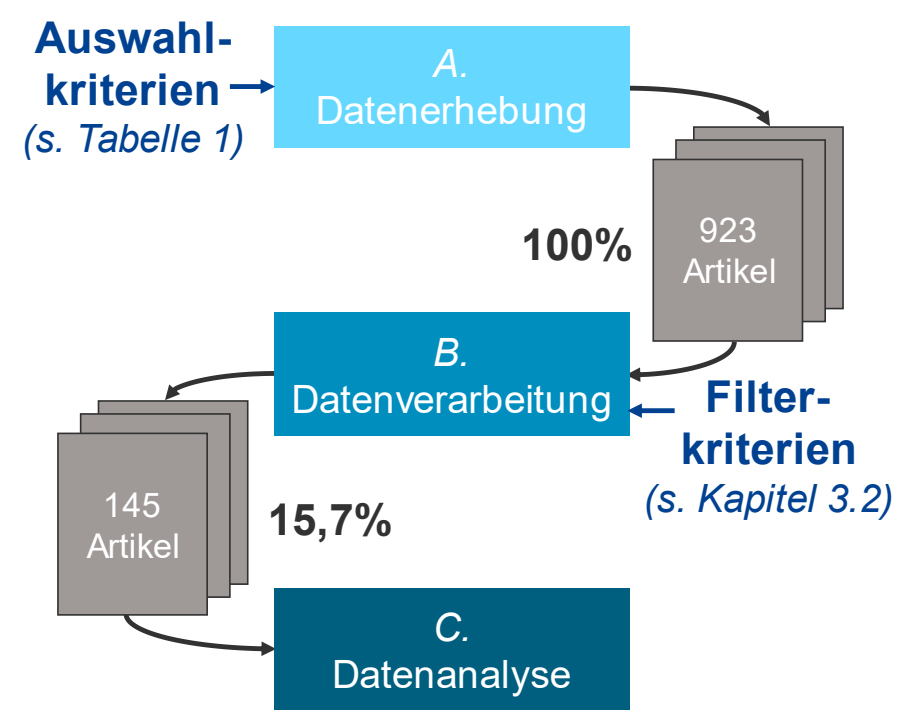

Abbildung 1: Methodik - 3-stufiger Prozess von der Datenerhebung bis zur Datenanalyse 
Tabelle 1: Kriterien für die Datenerhebung

\begin{tabular}{|l|l|}
\hline \multicolumn{1}{|c|}{ Eigenschaft } & \multicolumn{1}{c|}{ Kriterien } \\
\hline Plattform & ScienceDirect \\
\hline Veröffentlichungsjahre & 2013 - 2018 \\
\hline Suchphrase & $\begin{array}{l}\text { "Artificial Intelligence” UND } \\
\text { (,Application“ ODER „Fabrication“ ODER „Generation“ } \\
\text { ODER „Industry“ ODER „Production“ ODER „Industrial“ } \\
\text { ODER „Product“ ODER „Manufacturing“) }\end{array}$ \\
\hline Suchraum & Titel, Zusammenfassung, Schlagworte \\
\hline Veröffentlichungstyp & Research Articles \\
\hline Sprache & Englisch \\
\hline
\end{tabular}

\subsection{Datenerhebung}

Die in dieser Untersuchung verwendeten Daten stammen von einem der am weitesten verbreiteten Repositorien wissenschaftlicher Publikationen, der Plattform ScienceDirect. Als anfänglicher Suchbegriff wurde „Künstliche Intelligenz“ in Kombination mit zusätzlichen Schlüsselwörtern zu industriellen Anwendungen auf Veröffentlichungstitel, Zusammenfassungen und Stichwörter angewendet. Diese Begrenzung des Suchraums hatte den Zweck, die beiläufige Nennung der Suchwörter im Textkörper (bspw. in Form von Vergleichen und Abgrenzungen) herauszufiltern. Darüber hinaus wurden die Publikationsjahre auf 2013 bis 2018 begrenzt, um die Relevanz der betrachteten Veröffentlichungen zu erhöhen und sich auf aktuelle Entwicklungen zu konzentrieren. Um die unterschiedlichen Zahlen der verschiedenen Publikationsjahre vergleichen zu können, konnte das noch nicht abgeschlossene Jahr 2019 nicht berücksichtigt werden. Schließlich wurden nur englischsprachige Publikationen aufgenommen und der Artikeltyp musste „Research Article“, also ein die Ergebnisse originärer Forschung darstellender Artikel, sein, um Doppelnennungen durch Surveys, die bereits gesammelte Informationen erneut erwähnen, zu vermeiden. Tabelle 1 enthält eine Zusammenfassung der oben genannten Datenerhebungskriterien.

Die Datenerhebung lieferte insgesamt 923 verschiedene Artikel. Dies entspricht $100 \%$ der gesammelten Veröffentlichungen.

\subsection{Datenverarbeitung}

Der für diese Untersuchung erhobene Datensatz wurden anschließend aufbereitet, um noch enthaltene irrelevante Veröffentlichungen herauszufiltern. Zu diesem Zweck wurden die Veröffentlichungen manuell nach mehreren Kriterien gefiltert:

- Eine Veröffentlichung soll (eine) spezifische KI-Methode(n) im Fokus haben. 
- Eine Veröffentlichung soll die Nutzung der Methode(n) in Bezug auf einen spezifischen Anwendungsfall aus der Industrie (gemäß der Definition in Kapitel II) im Fokus haben.

- Eine Veröffentlichung soll eine Bewertung der Methode(n) in Bezug auf den Anwendungsfall beinhalten.

Dies ergab 145 unterschiedliche Artikel (was 15,7\% der gesammelten Veröffentlichungen entspricht), die alle oben genannten Anforderungen erfüllen und sich damit für die Ableitung von Aussagen über Anwendungsfälle und Methoden künstlicher Intelligenz in der anwendungsorientierten Forschung im Kontext von Industrie 4.0 eignen.

\subsection{Datenanalyse}

Die im Rahmen dieser Untersuchung verarbeiteten Daten wurden anschließend manuell analysiert. Zu diesem Zweck wurde jede Veröffentlichung basierend auf ihrem Volltext in Bezug auf unterschiedliche Dimensionen kategorisiert. In dieser Veröffentlichung werden lediglich die in Tabelle 2 aufgeführten Dimensionen näher betrachtet. Zwar wurden im Rahmen der hier vorgestellten Untersuchung weitere Dimensionen analysiert, jedoch lieferte dies keine ausreichend aussagekräftigen Ergebnisse.

Tabelle 2: Veröffentlichungsdimensionen und die dazugehörigen Ausprägungen

\begin{tabular}{|l|l|}
\hline \multicolumn{1}{|c|}{ Dimension } & \multicolumn{1}{c|}{ Ausprägung } \\
\hline Anwendungsfall & $\begin{array}{l}\text { Erkennung } \\
\text { Filterung } \\
\text { Modellbildung } \\
\text { Optimierung } \\
\text { Prognostik } \\
\text { Sortierung } \\
\text { Überwachung }\end{array}$ \\
\hline Datentyp & $\begin{array}{l}\text { Simulationsdaten } \\
\text { Experimentaldaten } \\
\text { Produktivdaten }\end{array}$ \\
\hline Herkunftsland & <Herkunftsland> \\
\hline Industriezweig & $\begin{array}{l}\text { Produktion } \\
\text { Chemie (Prozessindustrie) } \\
\text { Energie } \\
\text { Sonstige }\end{array}$ \\
\hline KI-Methoden- & $\begin{array}{l}\text { Fuzzy Systeme (Fuzzy) (Alcala-Fdez und Alonso 2016) } \\
\text { Künstliche Neuronal Netze (KNN) (Schmidhuber 2015) } \\
\text { Metaheuristiken (Metah.) (Glover und Kochenberger 2006) } \\
\text { Statistische Klassifizierer (Klass.) (Fukunaga 2013) } \\
\text { Support Vector Maschinen (SVM) (Salcedo-Sanz et al. 2014) } \\
\text { Sonstige }\end{array}$ \\
\hline Zitationen & <Anzahl> \\
\hline
\end{tabular}


Außerdem mussten aufgrund zu geringer Fallzahlen einige der ursprünglich erhobenen Ausprägungen unter „Sonstige“ zusammengefasst werden (s.u.). Sie werden vollständigkeitshalber in den Diagrammen mit abgebildet aber nicht weiter analysiert oder diskutiert.

Die Ausprägungen der Dimension „Anwendungsfall“ stellen das Ergebnis einer inhaltlichen Cluster-Bildung angelehnt an Niggemann et al. (2017) und Bitkom e.V. (2018) dar. Dabei wurden die Ausprägungen folgendermaßen definiert:

- Unter „Erkennung“ seien Anwendungsfälle zu verstehen, in denen es um die automatische, optische Erkennung und Interpretation von Texten, Objekten oder Abbildungen geht.

- Unter „Filterung“ seien Anwendungsfälle zu verstehen, in denen es um automatische Signalverarbeitung geht.

- Unter „Modellbildung“ seien Anwendungsfälle zu verstehen, in denen es um die automatische Parametrierung vorhandener bzw. die automatische Erstellung neuer Modelle geht.

- Unter „Optimierung“ seien Anwendungsfälle zu verstehen, in denen es um die automatische Optimierung einzelner Parameter oder ganzer Prozesse, bspw. in Form einer Anpassung der Prozessabfolge, geht.

- Unter „Prognostik“ seien Anwendungsfälle zu verstehen, in denen es um die automatische Prognose von Signalverläufen in der Zukunft oder, wie bspw. in Softsensoren, Gegenwart geht.

- Unter „Sortierung“ seien Anwendungsfälle zu verstehen, in denen es um die automatische Cluster-Bildung oder Klassifizierung, also bspw. die Einteilung in Fallgruppen, geht.

- Unter „Überwachung“ seien Anwendungsfälle zu verstehen, in denen es um die automatische Überwachung einzelner Signal- oder ganzer Prozessverläufe geht.

Die Dimension „Anwendungsfall“ wurde dabei nur für Artikel mit dem Industriezweig „Produktion" erhoben.

Die Ausprägungen der Dimension „Herkunftsland“ ergibt sich aus den in den jeweiligen Artikeln angegebenen Wirkungsstätten der Autorinnen und Autoren (nicht beschränkt auf Erstautorinnen und -autoren). 
Die Ausprägungen der Dimensionen „Industriezweig“ und „Datentyp“ stellen das Ergebnis einer inhaltlichen Cluster-Bildung dar. Dabei wurden in Bezug auf die Dimension "Industriezweige" die Ausprägungen "Bau", "Biotechnologie", "Landwirtschaft", "Logistik" sowie "Öl und Gas" unter "Sonstige" zusammengefasst.

Die Ausprägungen der Dimension „KI-Methoden-Gruppe“ ergeben sich aus den bezeichneten Quellen. Dabei wurden primär die Ausprägungen „Expertensysteme“, „Statistische Regression“ sowie „Numerische Analyse“ unter "Sonstige” zusammengefasst.

Die Ausprägungen der Dimension „Zitationen“ ergibt sich aus einer am 20.08.2019 durchgeführten Erhebung der Zitationen der einzelnen Artikel auf der Plattform GoogleScholar.

Als primäres Qualitätskriterium im Sinne der SLM wurde in dieser Untersuchung der Datentyp gewählt, da dieser Auskunft über die Anwendungsnähe der veröffentlichten Lösungen bzw. Methoden geben kann.

\section{Ergebnisse und Diskussion}

In diesem Abschnitt werden die Ergebnisse der Studie einem zweigeteilten Ansatz folgend dargestellt und diskutiert: Zunächst wird ein allgemeiner Überblick über Methoden der künstlichen Intelligenz in verschiedenen Industriezweigen gegeben. Anschließend wird ein fokussierter Blick auf die Situation im Industriezweig Produktion geworfen.

\section{1. Überblick}

Abbildung 2 zeigt die Entwicklung der Anzahl von Artikeln mit Bezug zur Anwendung von Methoden der künstlichen Intelligenz nach Industriezweig und Publikationsjahr. Es zeigt sich, dass die betrachteten Industriezweige einen erheblichen Anstieg der Anzahl einschlägiger Artikel insbesondere von 2017 nach 2018 erfahren (+85\% für Produktion, + 700\% für Chemie und $+433 \%$ für Energie). Der Anteil von Anwendungsfällen aus der Produktion beträgt insgesamt 44\% (Chemie: 10\%, Energie: 17\%, Sonstige: 29\%).

Das erst in jüngerer Vergangenheit vermehrte Auftreten einschlägiger Artikel mag aufgrund der bereits deutlich länger (siehe Einleitung) dominierenden Rolle des Themas in der wissenschaftlichen Gemeinschaft verwundern, jedoch ist zu bedenken, dass die Entwicklung marktreifer Lösungen auf Basis neuartiger Technologien eine erhebliche Zeit in Anspruch nimmt (Mankins 2009). Ein erster Ansatz zur Deutung dieser Verteilung der Industriezweige könnte 


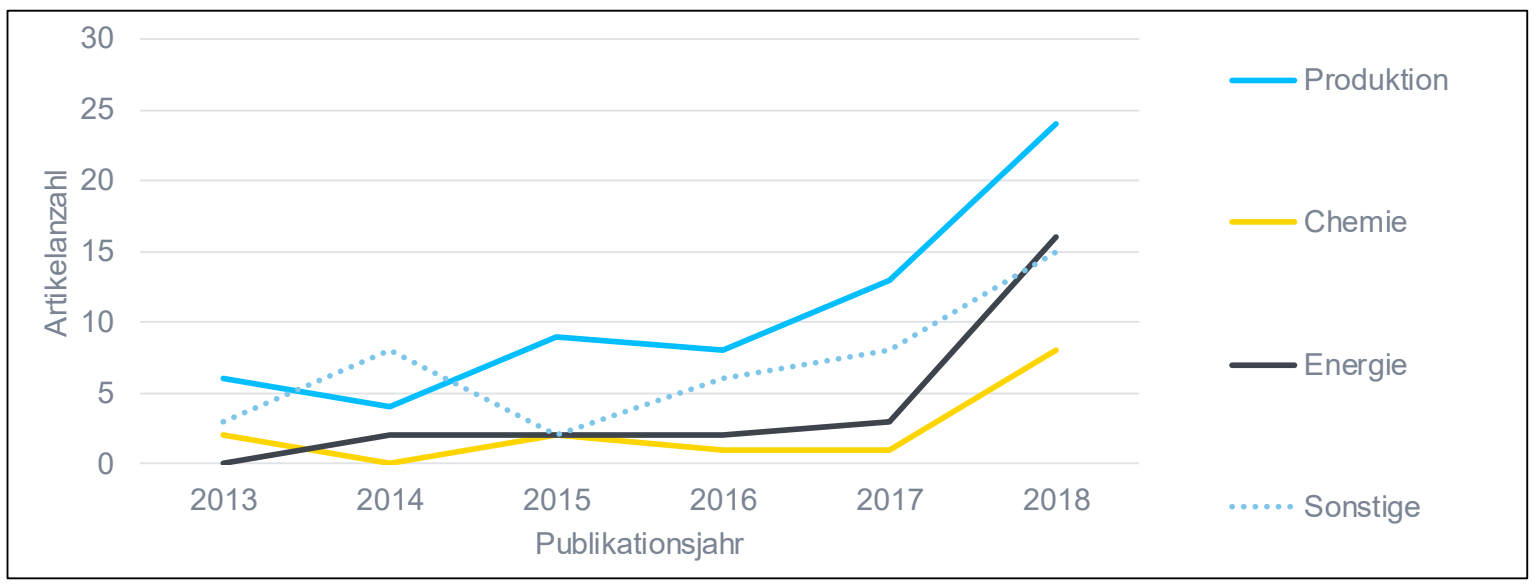

Abbildung 2: Entwicklung der Anzahl von Artikeln mit Bezug zur Anwendung von Methoden der künstlichen Intelligenz nach Industriezweig und Publikationsjahr

die häufig geringere Distanz zwischen Produkt und zu dessen Fertigung genutzter Werkzeuge in der Produktion gegenüber bspw. der Chemie sein.

Abbildung 3 zeigt die Entwicklung der Anzahl von Artikeln mit Bezug zur Anwendung von Methoden der künstlichen Intelligenz nach KI-Methoden-Gruppe und Publikationsjahr. Es zeigt sich, dass nennenswertes Wachstum für die meisten der betrachteten KI-Methoden-Gruppen erst 2017 vorliegt (Fuzzy: $+57 \%$, KNN: $+225 \%$, Metah.: $+33 \%$, Klass.: $+360 \%$, SVM: $+367 \%$, Sonstige: $+333 \%$ ). Lediglich KNN (Durchschnittliche jährliche Wachstumsrate 2013 bis 2017 : $+37 \%$ ) und Metah. (Durchschnittliche jährliche Wachstumsrate 2013 bis 2017: +52\%) zeigen auch vorher schon eine signifikant positive Entwicklung. Die absolute Zahl von KNN-Artikeln (65 Artikel 2018) übersteigt dabei die aller anderen Gruppen erheblich (zwischen 11 und 23 Artikeln 2018).

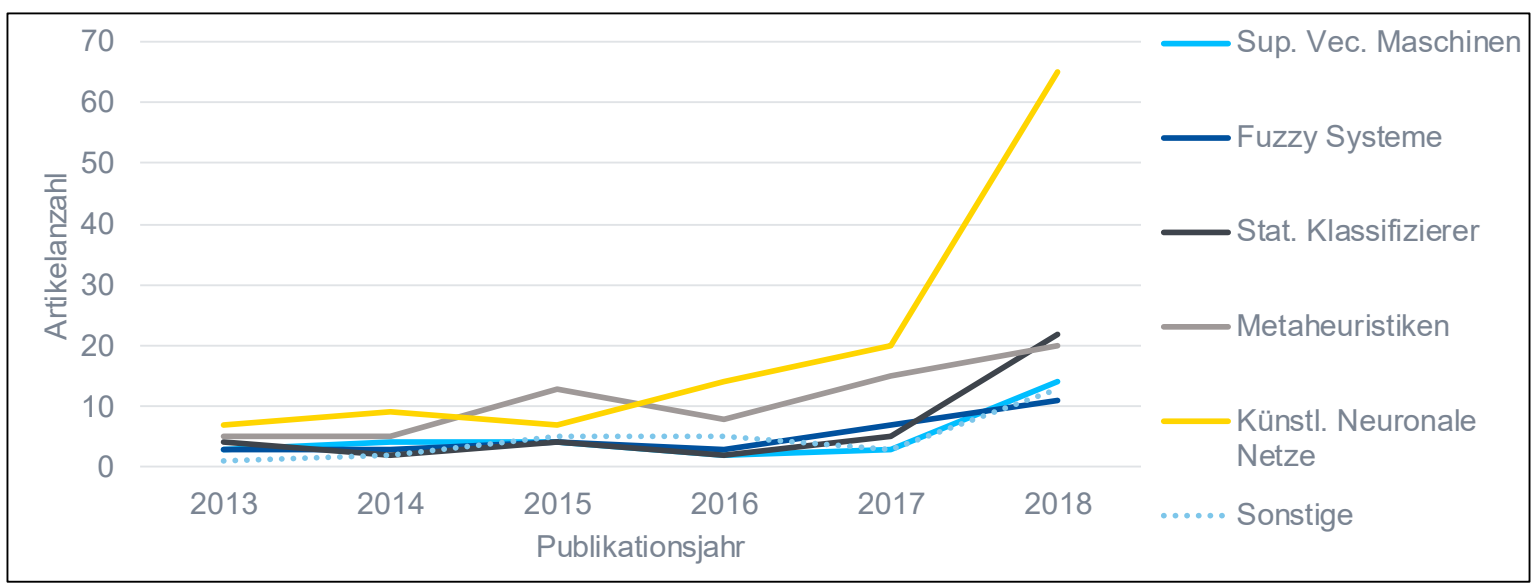

Abbildung 3: Entwicklung der Anzahl von Artikeln mit Bezug zur Anwendung von Methoden der künstlichen Intelligenz nach KI-Methoden-Gruppe und Publikationsjahr 


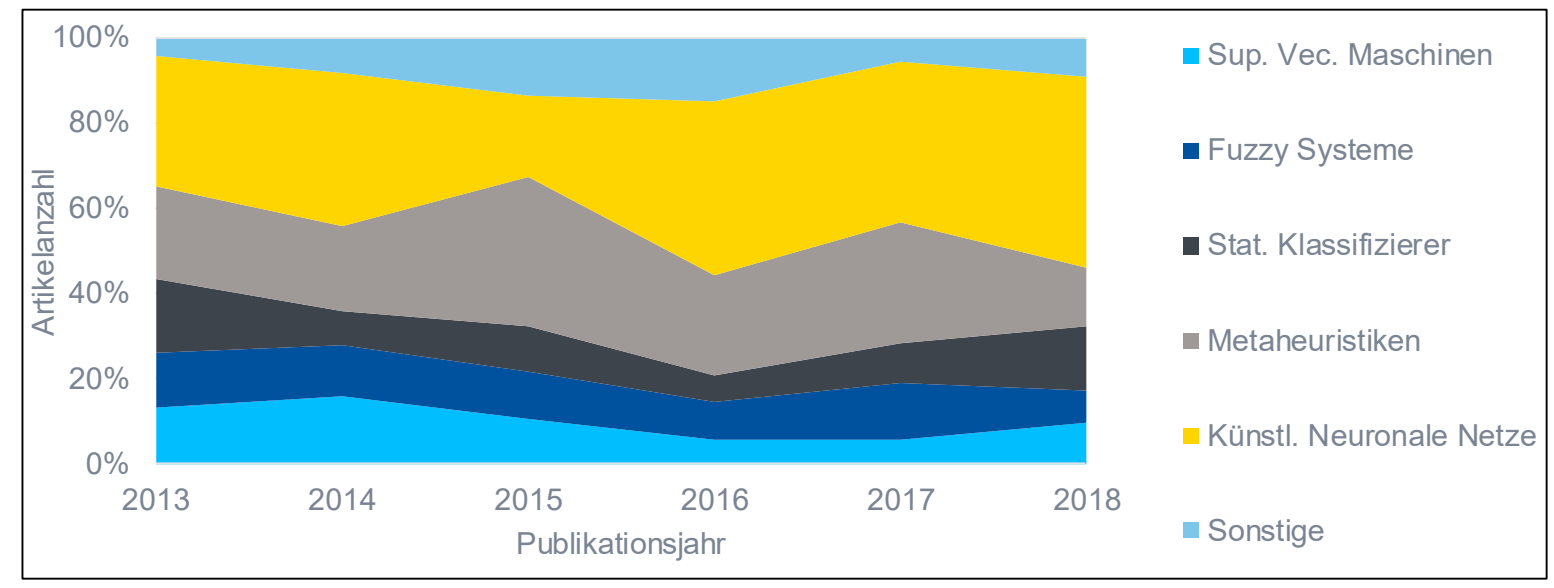

Abbildung 4: Entwicklung des Anteils von Artikeln mit Bezug zur Anwendung von Methoden der künstlichen Intelligenz nach KI-Methoden-Gruppe und Publikationsjahr

Verglichen mit der Gesamtzahl relevanter Artikel nahm der Anteil von KNN im Betrachtungszeitraum zu (von 30\% 2013 auf 45\% 2018, durchschnittlich 35\%), während insbesondere der Anteil von Fuzzy abnahm (von 13\% 2013 auf 8\% 2018, durchschnittlich 11\%, siehe auch Abbildung 4). Dies, genauso wie die absolute Verteilung, scheint in Einklang zu stehen mit den in den letzten zwei Jahrzehnten unter Nutzung von KNN erzielten Durchbrüchen in der KIForschung, die erhebliche Potentiale erkennen lassen und damit zu Experimenten auf derselben Basis einladen.

Abbildung 5 zeigt die Entwicklung der Anzahl von Artikeln mit Bezug zur Anwendung von Methoden der künstlichen Intelligenz nach genutztem Datentyp und Publikationsjahr. Es ist zu erkennen, dass die Nutzung von Simulationsdaten im Betrachtungszeitraum auf geringem Niveau ein leichtes Wachstum erfährt. Die Nutzung von Experimentaldaten verhält sich in den

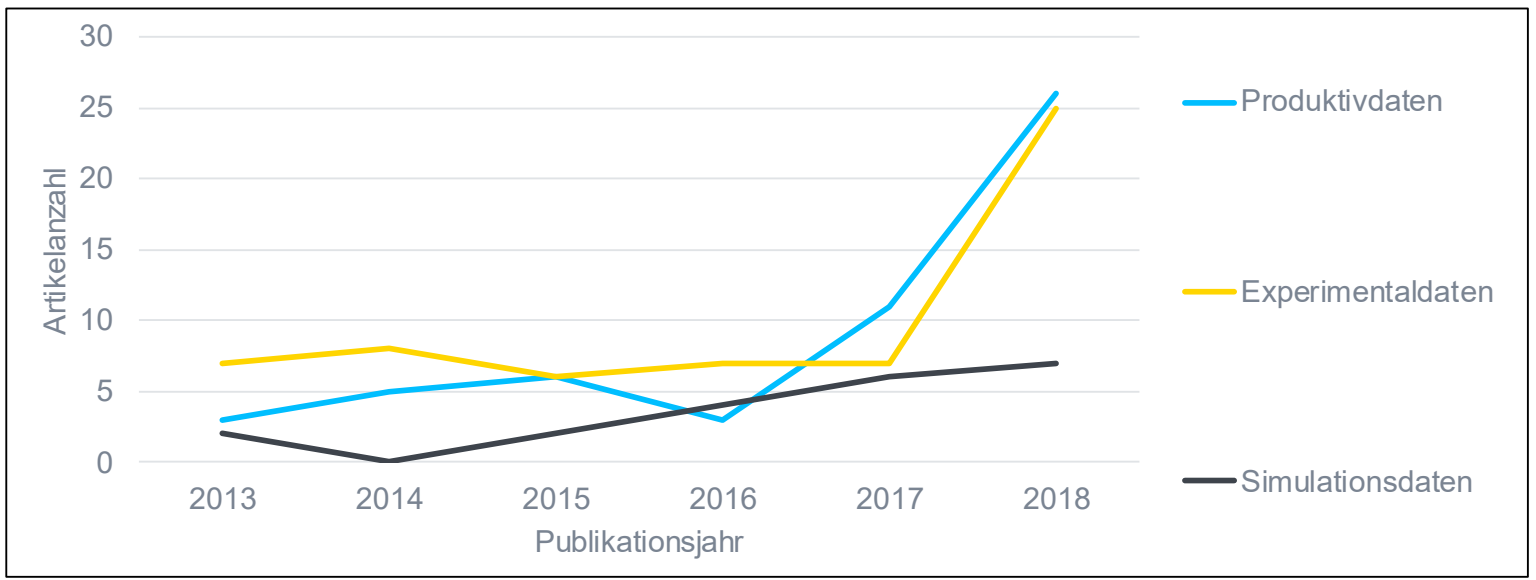

Abbildung 5: Entwicklung der Anzahl von Artikeln mit Bezug zur Anwendung von Methoden der künstlichen Intelligenz nach genutztem Datentyp und Publikationsjahr 
ersten 4 Jahren weitgehend statisch, um dann stark anzusteigen (+0\% von 2013 nach 2017, $+257 \%$ von 2017 nach 2018). Auch die Nutzung von Produktivdaten zeigt in den ersten Jahren einen statischen Verlauf und erst ab 2016 ein starkes Wachstum (+0\% von 2013 nach 2016, $+767 \%$ von 2016 nach 2018). In absoluten Zahlen liegen Experimentaldaten (60 Artikel) und Produktivdaten (54 Artikel) nahe beieinander und stellen damit - aufgrund der geforderten Anwendungsnähe nachvollziehbarerweise - die große Mehrheit (84\%) der Trainings- und Evaluationsdaten innerhalb der betrachteten Artikel. Verglichen mit der Gesamtzahl der Artikel nahm der Anteil der Produktivdaten-basierten Artikel im Betrachtungszeitraum stark zu (von 25\% 2013 auf 45\% 2018), während der Simulationsdaten (von 17\% auf 12\%) und Experimentaldaten (von 58\% auf 43\%) abnahm. Dies erscheint plausibel, da angewandte Forschung auf Basis neuer Technologien vielfach mit Simulations- und Experimentaldaten startet um Potentiale und Risiken abzuwägen und sich erst im weiteren Verlauf Produktivumgebungen und den dort erhobenen Daten zuwendet.

Abbildung 6 zeigt, dass die Potenziale der Methoden künstlicher Intelligenz für die Industrie bereits in jedem Kontinent entdeckt wurden und erforscht werden. Dabei ist deutlich zu erkennen, dass China und die USA am meisten Veröffentlichungen in diesem Bereich aufweisen. Werden nur die Länder der korrespondierenden Autoren betrachtet liegen Deutschland und Indien nur eine Veröffentlichung hinter China und den USA. Damit spiegelt sich in diesem

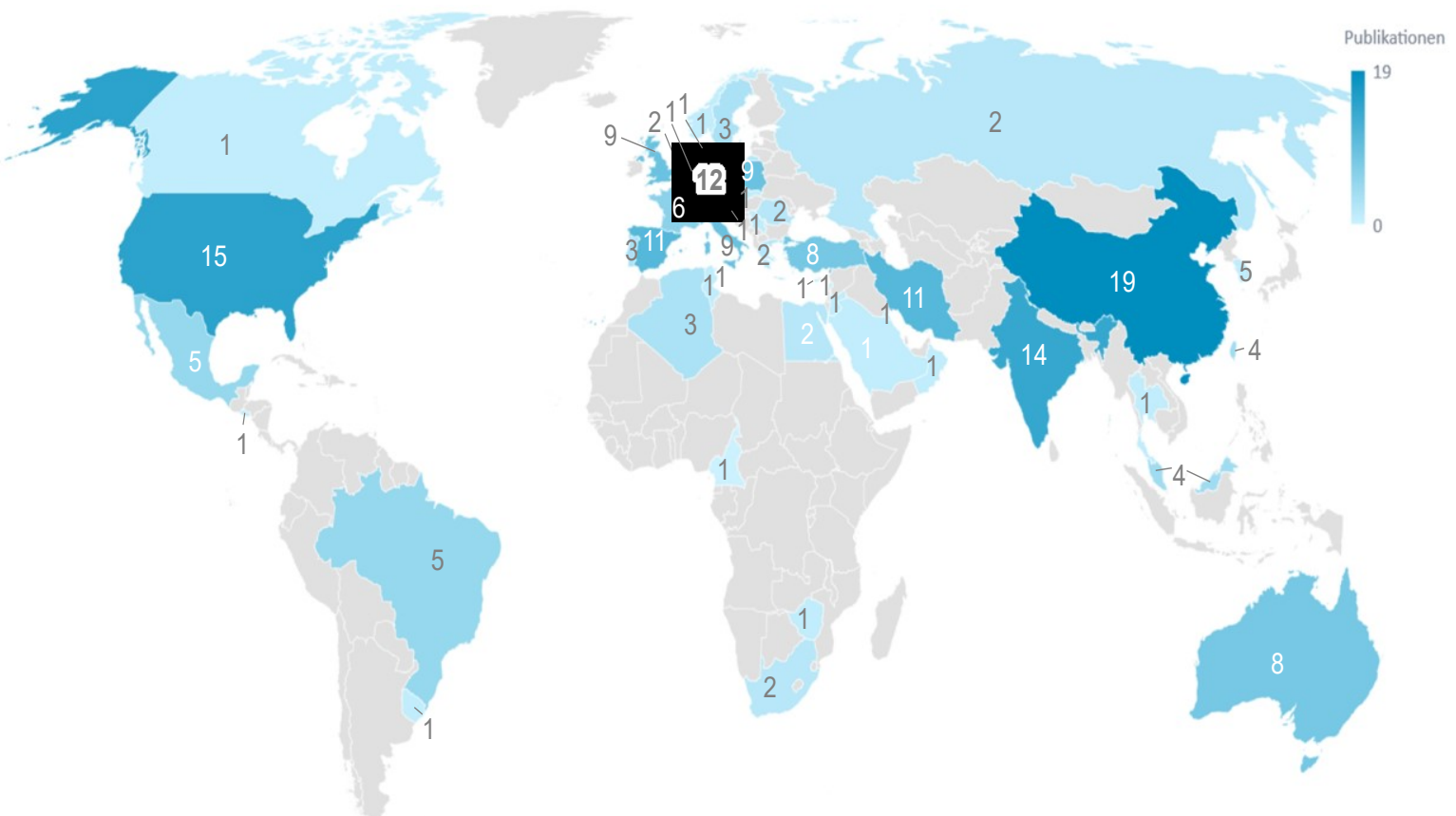

Abbildung 6: Anzahl von Artikeln mit Bezug zur Anwendung von Methoden der künstlichen Intelligenz nach Herkunftsland 
Beispiel genau der Wissenschaftswettbewerb im Bereich der Automatisierung und der Informatik der vier in diesen Bereichen führenden Länder wider.

\subsection{Künstliche Intelligenz in der Produktion}

Abbildung 7 zeigt die Entwicklung der Anzahl von Artikeln mit Bezug zur Anwendung von Methoden der künstlichen Intelligenz in der Produktion nach KI-Methoden-Gruppe und Publikationsjahr. Es zeigt sich, dass für die meisten KI-Methoden-Gruppen weder die Fallzahl signifikant ist noch ein eindeutiger Trend zu erkennen wäre. Lediglich KNN und Metah. weisen ein moderates (Metah.: +233\% von 2013 nach 2018) bis starkes (KNN: +360\% von 2013 nach 2018) Wachstum bei einer insgesamt aussagekräftigen Fallzahl auf. Vergleicht man Abbildung 7 mit Abbildung 3, die denselben Sachverhalt über alle Industriezweige hinweg abbildete, so fällt auf, dass der Anteil von Metah. größer (24\% insgesamt gegenüber $30 \%$ in Produktion) und das Wachstum von Klass. $(2018+245 \%$ verglichen mit dem Mittelwert insgesamt gegenüber $+125 \%$ verglichen mit dem Mittelwert in Produktion) sowie das von KNN insgesamt flacher $(2018+220 \%$ verglichen mit dem Mittelwert insgesamt gegenüber $+182 \%$ verglichen mit dem Mittelwert in Produktion) ist.

Trotzdem nahm der Anteil von KNN verglichen mit der Gesamtzahl relevanter Artikel im Betrachtungszeitraum stark zu (von 31\% 2013 auf 47\% 2018, durchschnittlich 34\%, siehe Abbildung 8). Die Anteile der anderen KI-Methoden-Gruppen lässt leider keine eindeutigen Trends erkennen. Auch in der Produktion ist somit der Trend hin zur Nutzung von KNN (siehe Erläuterungen zu Abbildung 4) zu erkennen.

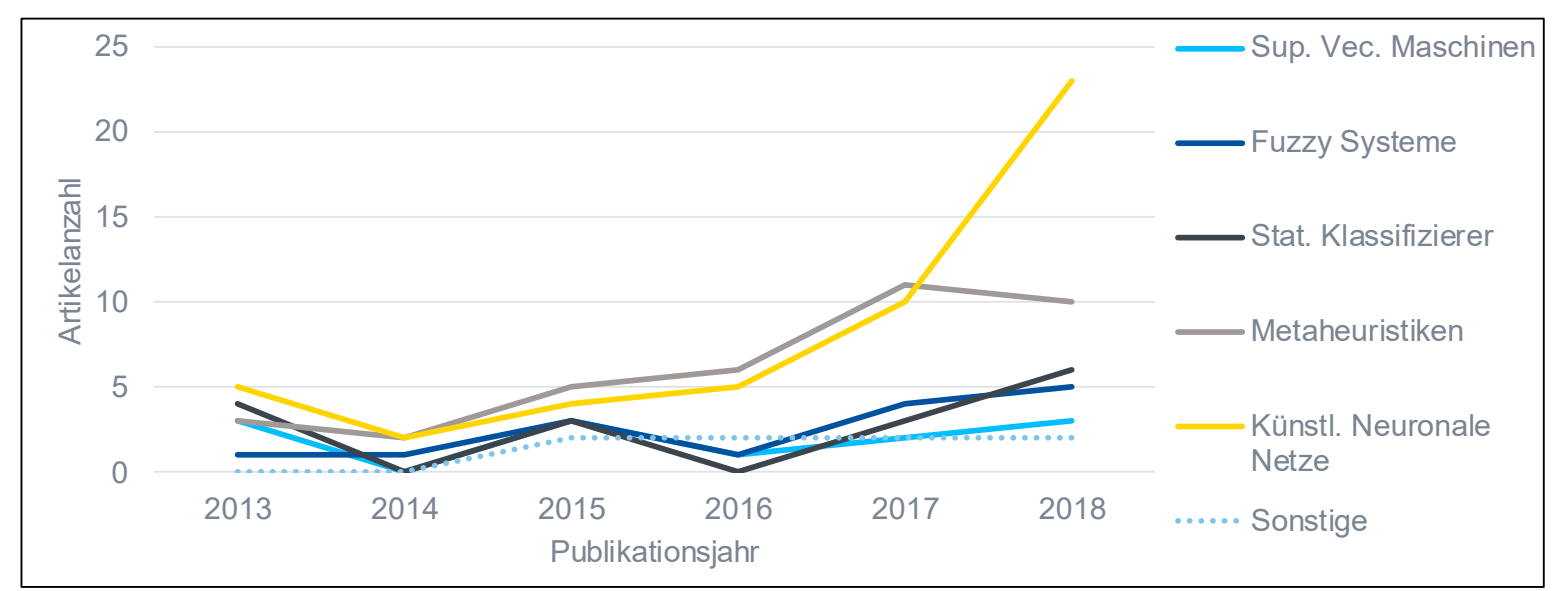

Abbildung 7: Entwicklung der Anzahl von Artikeln mit Bezug zur Anwendung von Methoden der künstlichen Intelligenz in der Produktion nach KI-Methoden-Gruppe und Publikationsjahr 


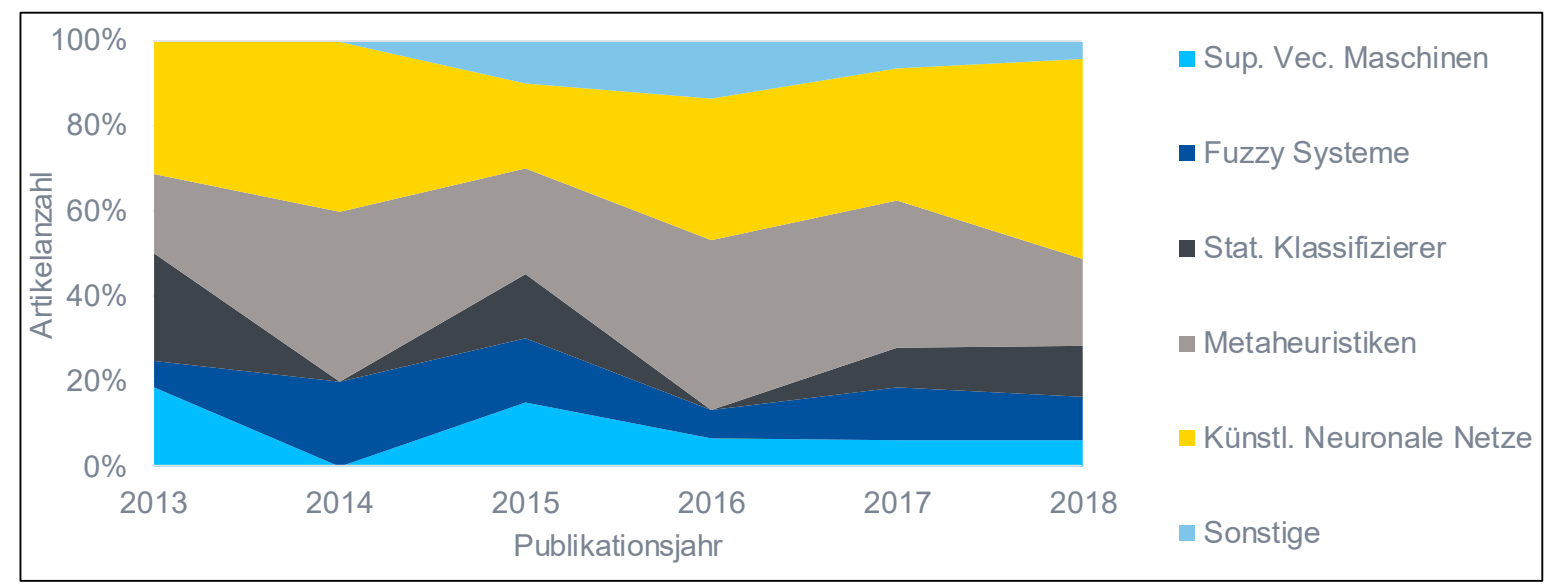

Abbildung 8: Entwicklung des Anteils von Artikeln mit Bezug zur Anwendung von Methoden der künstlichen Intelligenz in der Produktion nach KI-Methoden-Gruppe und Publikationsjahr

Abbildung 9 zeigt die Entwicklung der Anzahl von Artikeln mit Bezug zur Anwendung von Methoden der künstlichen Intelligenz in der Produktion nach genutztem Datentyp und Publikationsjahr. Es ist zu erkennen, dass die Nutzung von Simulationsdaten auf geringem Niveau ein leichtes Wachstum erfährt. Experimentaldaten weisen ein stagnierendes bis leicht abfallendes Level über die ersten vier Jahre des Betrachtungszeitraums und einen moderaten Anstieg im letzten Jahr auf (+125\% von 2017 nach 2018, +64\% gegenüber Mittelwert). Der Anteil von Produktivdaten hingegen stieg von 0\% in den Jahren 2013 und 2014 auf 48\% 2018. Ein Vergleich der Veränderungen der relativen Anteile, so auch der Abnahme des Anteils von Simulations- (von 25\% auf 13\%) und Experimentaldaten (von 75\% auf 39\%), in der Produktion mit denen insgesamt ergibt ein ähnliches Bild. Auffallend ist jedoch der späte Start der Nutzung von Produktivdaten in der Produktion (2015), der in anderen Industriezweigen schon vor 2013

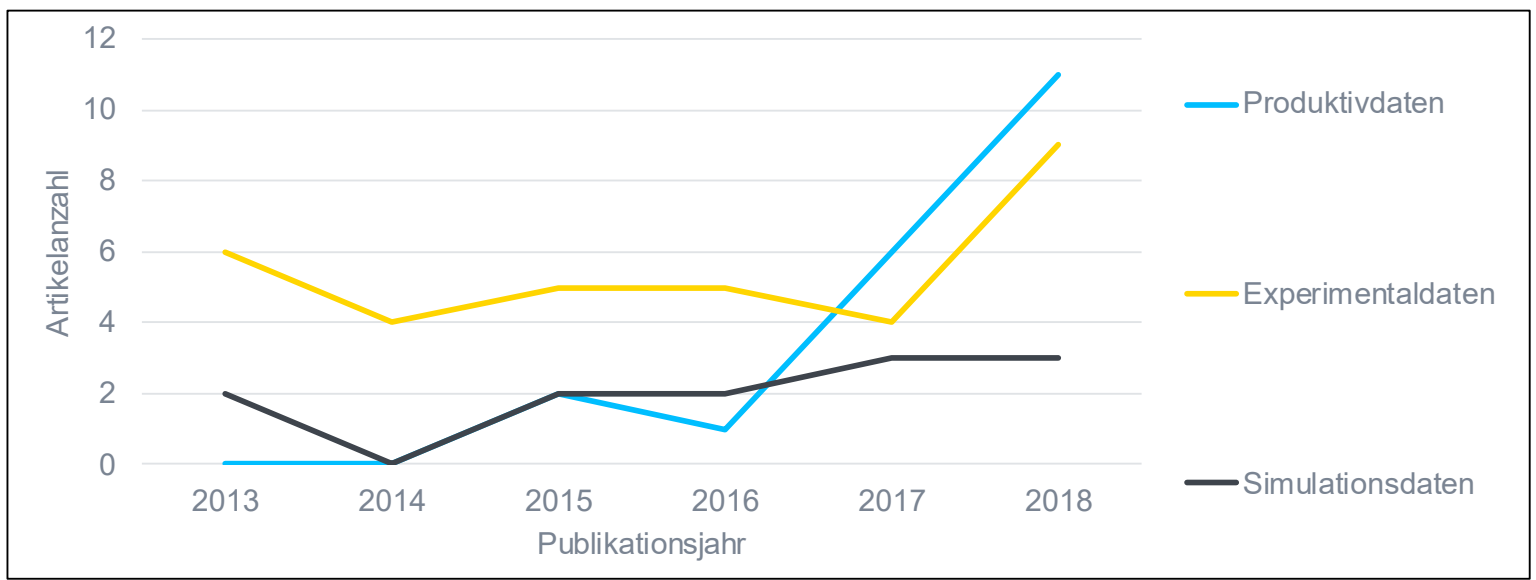

Abbildung 9: Entwicklung der Anzahl von Artikeln mit Bezug zur Anwendung von Methoden der künstlichen Intelligenz in der Produktion nach genutztem Datentyp und Publikationsjahr 
erfolgt sein muss. Dies ist insbesondere bemerkenswert, da die auf anderen Daten basierende, anwendungsnahe Forschung in der Produktion der in anderen Industriezweigen zeitlich voraus war (siehe oben).

Abbildung 10 zeigt die Verteilung der Anzahl von Artikeln mit Bezug zur Anwendung von Methoden der künstlichen Intelligenz in der Produktion nach Anwendungsfall. Es zeigt sich, dass in dem Anwendungsfall Optimierung die Publikationszahl am größten ist. Insbesondere zeigt sich hier eine stärkere Konzentration im Bereich der Parameteroptimierung mit 15 Veröffentlichungen zu 9 Veröffentlichungen bei der Prozessoptimierung. Vogel-Heuser (2011) schreibt, dass hoher Wettbewerbsdruck und steigende gesetzliche Vorgaben Unternehmen verstärkt dazu zwingen ihre Prozesse und Produkte zu optimieren, weshalb die starke Publikationstätigkeit hier nachvollziehbar ist. Zunächst erscheint es überraschend, dass die Sortierung an zweiter Stelle liegt. Jedoch ist hier auch die Fehlerdiagnose mit einbezogen, die anhand von Vergleichen zwischen historischen Fehlerfällen und aktuellem Systemverhalten eine Zuordnung versucht. Da Fehler einen hohen Kostentreiber bei der Produktion darstellen, ist den betreffenden Unternehmen viel daran gelegen diese Kosten mittels automatischer Fehlerdiagnose zu reduzieren. Auch die Zitationszahl, welche für die gesammelten Veröffentlichungen im Bereich der Fehlerdiagnose am höchsten von allen Veröffentlichungen im Industriezweig Produktion ist, spiegelt dies wider. In eine ähnliche Richtung gehen die Veröffentlichungen mit dem Anwendungsfall Prognostik. Diese werden durch den gleichen Kostenfaktor wie die

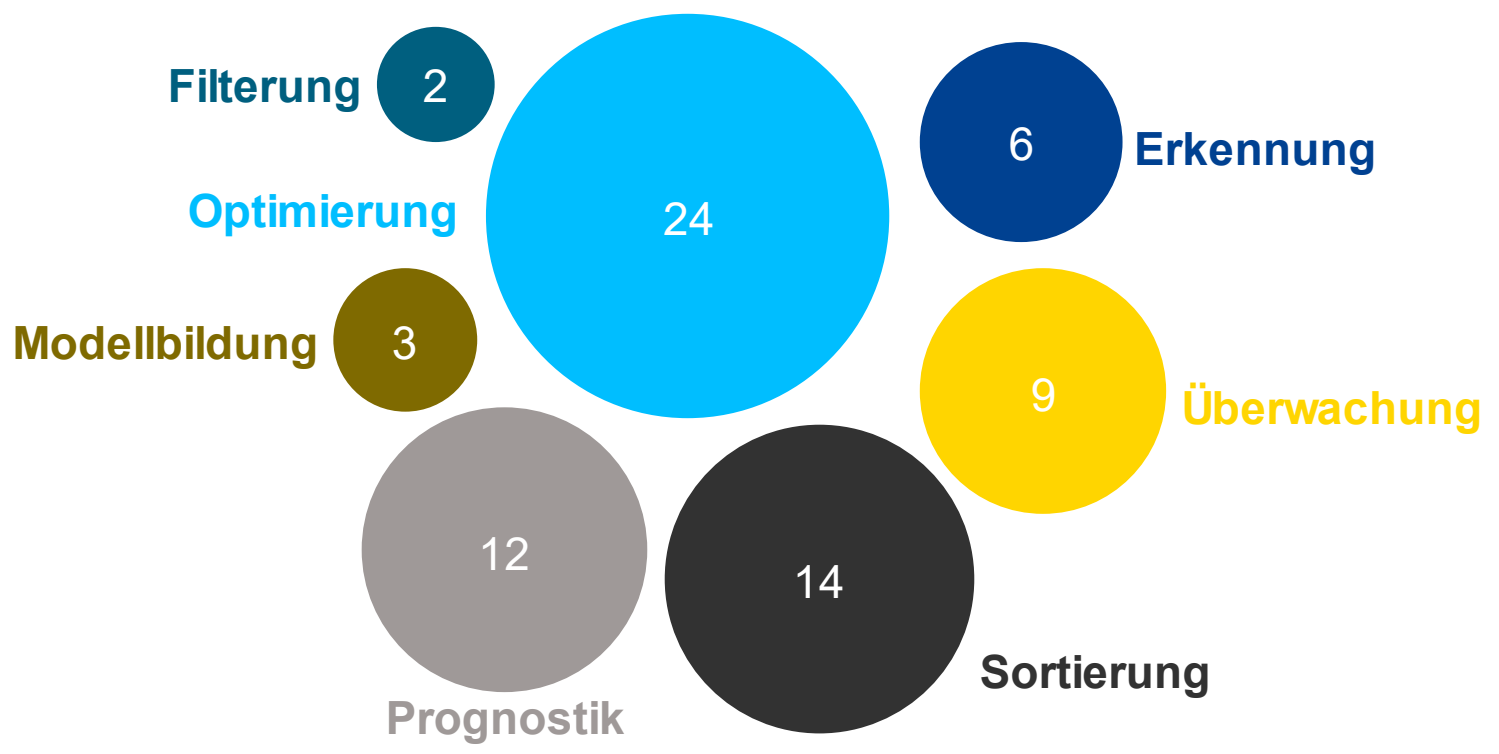

\footnotetext{
Abbildung 10: Verteilung der Anzahl von Artikeln mit Bezug zur Anwendung von Methoden der künstlichen Intelligenz in der Produktion nach Anwendungsfall
} 
Fehlerdiagnose getrieben und befinden sich auf dem dritten Rang der am stärksten publizierten Anwendungsfälle. Mit unter 10 Veröffentlichungen sind die Anwendungsfälle Überwachung, Erkennung, Modellbildung und Filterung bisher scheinbar weniger relevant.

\section{Fazit}

In dieser Veröffentlichung wurden 145 wissenschaftliche Artikel analysiert, die mittels einer an die Systematic Literature Review angelehnten Methode aus einer 923 einschlägige Publikationen von der Plattform ScienceDirect umfassenden Eingangsdatenmenge herausgefiltert wurden.

Es konnte anhand der Ergebnisse gezeigt werden, dass es in den Jahren 2013 bis 2018 einen stetigen Zuwachs der Publikationen über die Nutzung von Methoden künstlicher Intelligenz in allen betrachteten Industriezweigen gab. Hauptsächlich trug dabei ein Anstieg des Gebrauchs von neuronalen Netzen angewandt auf Produktivdaten in den jüngeren Jahren zu dem Zuwachs bei. Ein genauerer Blick auf die Publikationen im Industriezweig Produktion ergibt ein ähnliches Bild: Auch hier stieg die Anzahl an Veröffentlichung über das datengetriebene maschinelle Lernen stetig und im Besonderen aufgrund der Nutzung von künstlichen neuronalen Netzen. Auch hier zeigt sich ein Wechsel über die Zeit von Simulationsdaten hin zu Produktivdaten. Dies lässt auf eine zunehmende Reife der eingesetzten KI-Methoden und daraus folgend auf eine Zunahme des tatsächlichen Einsatzes derartiger Methoden in der industriellen Praxis schließen.

Innerhalb der einzelnen KI-Methoden-Gruppen herrscht eine große Vielfalt und Dynamik. Spezifische Methoden werden vielfach nur einmal angewandt, sodass sich insbesondere in der Gruppe der künstlichen neuronalen Netze kein Trend hin zu einer spezifischen Methode ablesen lässt. Ganz im Gegenteil scheint der Trend zu spezifisch auf den jeweiligen Anwendungsfall angepassten Methoden bzw. Algorithmen ungebrochen. Einzig die Komplexität, also die zunehmende sequenzielle Kombination verschiedener Algorithmen zur Erfüllung einer gemeinsamen Aufgabe, scheint zu steigen. Dies deckt sich mit der Entwicklung in der anwendungsferneren (Grundlagen-)Forschung.

Die Anwendungsfälle in der Produktion zeigen, dass die größte Anzahl an Publikationen die Parameter- und die Prozessoptimierung in diesem Industriezweig betreffen. Neben der Optimierung sind die Prognostik und die Sortierung mit der Fehlerdiagnose der Publikationsanzahl nach ein bedeutendes Forschungsfeld. Trotz der Konzentration entsprechender Publikationen 
auf diese Anwendungsfallkategorien werden Methoden künstlicher Intelligenz jedoch zunehmend über die gesamte Breite industrieller Anwendungsfälle eingesetzt.

Eine klare Zuordnung einzelner Methoden zu spezifischen Anwendungsfällen ergibt sich nicht. Vielmehr legt die große Vielfalt der erfolgreich angewandten Ansätze den Schluss nahe, dass eine solche auch prinzipiell nicht möglich ist. Bestimmend für die Auswahl konkreter KI-Methoden sind vielmehr Projekteigenschaften (Datenverfügbarkeit, Datenstrukturen etc.), Umweltbedingungen (Systemtopologien, verfügbare Hard- und Softwareumgebungen etc.) und grundlegende Charakteristika der jeweiligen KI-Methoden (Trainingsaufwand, Speicherbedarf etc.). Dies bedeutet in der Praxis, dass bspw. nicht die optimale Angepasstheit eines KNNAlgorithmus an ein Optimierungsproblem ausschlaggebend für dessen Projekteignung ist, sondern Eigenschaften wie bspw. die Verfügbarkeit entsprechender Bibliotheken für das Zielsystem, die Kompatibilität mit eventuell vorhandenen Hardwarebeschleunigern oder der resultierende Kommunikationsaufwand zur Bereitstellung der benötigten Daten. Umgekehrt folgt daraus, dass bei einem gegebenen Projekt bereits vergleichsweise kleine Änderungen von Projekteigenschaften oder Umweltbedingungen zu ganz unterschiedlichen Auswahlentscheidungen bzgl. der zu verwendeten KI-Methode führen können - ohne die Lösungsqualität zu verschlechtern.

Hesenius et al. (2019) tragen diesem Umstand mit ihrem Ansatz eines an die Entwicklung von datengetriebenen Anwendungen angepassten Software-Entwicklungskonzepts Rechnung. Die Nutzung datengetriebener Methoden künstlicher Intelligenz erfordert eine Anpassung des aktuellen Entwicklungsprozesses, denn die Auswahl konkreter KI-Methoden erfordert ein iterativ explorierendes Verfahren und lässt sich nicht im Vorhinein aufgrund einiger weniger Annahmen bspw. bezüglich des allgemeinen Anwendungsfalls sinnvoll vornehmen.

\section{Open Data}

Im Sinne des Open Data Konzepts sowie zur Ermöglichung der Überprüfung ihrer Ergebnisse stellen die Autoren den hier vorgestellten, 145 Artikel umfassenden Datensatz dauerhaft unter CC BY-SA 4.0 auf https://www.ias.uni-stuttgart.de/dokumente/Dataset_Utilization_Industry2019.zip zur Verfügung.

\section{Literatur}

Alcala-Fdez J, Alonso JM (2016) A Survey of Fuzzy Systems Software: Taxonomy, Current Research Trends, and Prospects. IEEE Trans. Fuzzy Syst., 24.1:40-56 
Bundesverband Informationswirtschaft, Telekommunikation und neue Medien e. V (Bitkom e.V.). (2018) Digitalisierung gestalten mit dem Periodensystem der Künstlichen Intelligenz Ein Navigationssystem für Entscheider. Berlin

Diez-Olivan A, Del Ser J, Galar D, Sierra B (2019) Data fusion and machine learning for industrial prognosis: Trends and perspectives towards Industry 4.0. Information Fusion 50:92111

Flasiński M (2016) Introduction to artificial intelligence. 1. Aufl., Springer, Wiesbaden

Fukunaga K (2013) Introduction to statistical pattern recognition. Elsevier, Amsterdam

Glover FW, Kochenberger GA (Hrsg) (2006) Handbook of metaheuristics. 57. Ausgabe, Springer, Wiesbaden

Hesenius M, Schwenzfeier N, Meyer O, Koop W, Gruhn V (2019) Towards a Software Engineering Process for Developing Data-Driven Applications. In Proc. 7th Int. Workshop on Realizing Art. Int. Synergies in Softw. Eng. IEEE Press, 35-41

Jeschke S, Brecher C, Meisen T, Özdemir D, Eschert T (2017) Industrial internet of things and cyber manufacturing systems. In: Jeschke S, Brecher C, Song H, Rawat DB (Hrsg) Industrial Internet of Things. Springer, Berlin und Heidelberg, 9-13

Jordan MI, Mitchell TM (2015) Machine learning: Trends, perspectives, and prospects. Science 349.6245:255-260

Kagermann H, Wahlster W, Helbig J (2013) Umsetzungsempfehlungen für das Zukunftsprojekt Industrie 4.0. Abschlussbericht des Arbeitskreises Industrie 4.0

Kitchenham B (2004) Procedures for performing systematic reviews. Technical Report der Keele University, Keele

Mankins JC (2009) Technology readiness assessments: A retrospective. Acta Astronautica 65.9-10:1216-1223

Muhuri PK, Shukla AK, Abraham A (2019) Industry 4.0: A bibliometric analysis and detailed overview. Engineering Applications of Artificial Intelligence, 78:218-235 
Niggemann O, Biswas G, Kinnebrew JS, Khorasgani H, Voglmann S, Bunte A (2017) Datenanalyse in der intelligenten Fabrik. In: Vogel-Heuser B, Bauernhansl T, ten Hompel M (Hrsg) Handbuch Industrie 4.0 Bd. 2, Springer Vieweg, Berlin und Heidelberg, S 471-490

Runkler TA (2015) Data Mining-Methoden und Algorithmen intelligenter Datenanalyse. 2. Aufl. Vieweg+Teubner, Wiesbaden

Salcedo-Sanz S, Rojo-Álvarez JL, Martínez-Ramón M, Camps-Valls G (2014) Support vector machines in engineering: an overview. WIREs Data Mining Knowl Discov, 4.3:234-267

Schmidhuber J (2015) Deep learning in neural networks: an overview. Neural Networks, 61:85-117

Sharp M, Ak R, Hedberg T (2018) A survey of the advancing use and development of machine learning in smart manufacturing. J. o. Manu. Sys. 48:170-179

Vogel-Heuser B (2011) Erhöhte Verfügbarkeit und transparente Produktion. In: Tagungsband Automation Symposium, Kassel University Press, Kassel

Vogel-Heuser, B (2017) Herausforderungen und Anforderungen aus Sicht der IT und der Automatisierungstechnik. In: Vogel-Heuser B, Bauernhansl T, ten Hompel M (Hrsg) Handbuch Industrie 4.0 Bd. 4, Springer Vieweg, Berlin und Heidelberg, S 33-44

Wang J, Ma Y, Zhang L, Gao RX, Wu D (2018) Deep learning for smart manufacturing: Methods and applications. J. o. Manu. Sys., 48:144-156 